\title{
Criminologie
}

\section{Autour de Michel Foucault et de son oeuvre. Bibliographie}

Volume 26, numéro 1, 1993

Michel Foucault et la (post) modernité

URI : https://id.erudit.org/iderudit/017332ar

DOI : https://doi.org/10.7202/017332ar

Aller au sommaire du numéro

Éditeur(s)

Les Presses de l'Université de Montréal

ISSN

0316-0041 (imprimé)

1492-1367 (numérique)

Découvrir la revue

Citer ce document

(1993). Autour de Michel Foucault et de son oeuvre. Bibliographie.

Criminologie, 26(1), 123-131. https://doi.org/10.7202/017332ar d'utilisation que vous pouvez consulter en ligne.

https://apropos.erudit.org/fr/usagers/politique-dutilisation/ 


\section{A. EUVRES CHOISIES DE MICHEL FOUCAULT}

Maladie mentale et personnalisé, Paris, Presses Universitaires de France, 1954.

Maladie mentale et psychologie, Paris, Presses Universitaires de France, 1962, 1966. Il s'agit d'une édition revue du précédent comportant une nouvelle seconde partie, ainsi qu'une nouvelle conclusion.

«Les déviations religieuses et le savoir médical ", Hérésies et sociétés dans l'Europe pré-industrielle $11^{e}-18^{e}$ siècles. Communications et débats du Colloque de Royaumont présentés par Jacques LeGoff, Paris, Mouton, 1968, p. 19-29.

«Un si cruel savoir», Critique 182 (juillet 1962) p. 597-611. Un essai sur Les égare. ments du cơur et de l'esprit de Claude Crébillon et Pauliska ou la perversité moderne de J.A. Reveroni de Saint-Cyr.

"Préface d̀ la transgression", Critique 195-196 (août-septembre 1963), p.751-769.

«Distance, aspect, origine», Critique 198 (novembre 1963), p.931-945.

«Nietzsche, Freud, Marx m, Cahiers de Royaumont 6: Nietzsche, Paris, Éditions de Minuit, 1967, p. 183-200.

«C'était un nageur entre deux mots», Arts-loisirs 54 (oct. 5, 1966) p. 8-9. Entrevue avec Claude Bonnefoy au sujet d'André Breton.

"La pensée médicale », Le concours médical 88 (22 octobre 1966), p. 6285-6286.

"Deuxième entretien: Sur les façons d'écrire l'histoire", Les lettres françaises 1187 (15 juin 1967), p.6-9. Entretien avec Raymond Bellour. Publié aussi dans: R. Bellour, Le livre des autres. Paris, Éditions de l'Herne, 1971, p. 189-207.

«Foucault répond à Sartre», La Quinzaine littéraire 46 (1-15 mars 1968), p. 20-22. Interview avec Jean-Pierre El Kabach. La publication de la transcription de cet entretien avec Jean-Pierre El Kabbach a suscité une réplique acerbe de Michel Foucault parue dans le numéro 15-31 sous le titre «Une mise au point de Michel Foucault m.

«Réponse à une question», Esprit 371 (mai 1968) p. 850-874.

"Réponse au Cercle d'épistémologie», Cahiers pour l'analyse 9: Généalogie des sciences (été 1968) p.9-40.

1. Bibliographie inspirée de celle de James Bernauer et de David Rasmussen dans The Final Foucaul, Cambridge, Mass., MIT Press, 1988 et complétée par JeanPaul Brodeur, Centre international de criminologie comparée, Université de Montréal. 
«Médecins, juges et sorciers au XVII ${ }^{e}$ siècle \#, Médecine de France 200, (1969) p. 121 128.

"Qu'est-ce qu'un auteur?", Bulletin de la Société française de phitosophie 63 (juilletseptembre 1969), p.73-104. Les commentaires de Michel Foucault sont suivis d'une discussion avec Maurice de Gandillac, Lucien Goldmann, Jacques Lacan, Jean d'Ormesson, J. Ullmo et Jean Wabl.

«Le piège de Vincennes», Le Nouvel Observateur 274 (9 février 1970), p.33-35. Une entrevue avec Patrick Loriot.

«II y aura scandale, mais.. ", Le Nouvel Observateur 304 (7 septembre 1970), p. 40. Un mot sur Eden, Eden, Eden de Pierre Guyotat.

«Nietzsche, la gennéalogie, l'histoire», in Hommage à Jean Hyppolite, Paris, Presses Universitaires de France, 1971, p. 145-172.

«A Conversation with Michel Foucault», Partisan Review 38 (1971), p. 192-201. Une entrevue de John Simon.

«Human Nature: Justice versus Power», in Reflexive water: The Basic Concerns of Mankind. Sous la direction de Fons Elders, London, Souvenir Press, 1974, p. 134197. Une discussion entre Michel Foucault et Noam Chomsky.

«Par delà le bien et le mal », Actuel 14 (novembre 1971), p.42-47. Entretien avec M.A. Burnier et P. Graine. Rédité.

"Monstrosities in Criticism», Iraduit par Robert J. Matthews. Diacritics I, 1 (automne 1971), p. 57-60. Réplique à l'article «The Order of Things» par George Steiner: "The mandarin of the bour - Michel Foucault». The New York Times Book Review, 8 (28 février 1971), p. 23-31.

«Foucault responds 2 », Diacritics I, 2 (hiver 1971) p. 60. La réplique de Foucault à la réponse de Steiner.

"Sur la justice populaire: Débat avec les maos», Les Temps modernes 310 (1972), p. 335-366. Une discussion avec Philippe Gavi et Pierre Victor.

Interviews and Other Writings, 1972-1977. Sous la direction de Colin Gordon, New York, Pantheon, 1980, p. 1-36.

"Les intellectucls ct le pouvoir", L'arc 49 (1972), p.3-10. Une conversation entre Michel Foucault et Gilles Deleuze qui a eu lieu le 4 mars 1972.

«Michel Foucault on Attica», Telos 19 (1974), p.154-161. Une entrevue avec John Simon en 1972.

"Table ronde», Esprit 413 (avril/mai 1972), p.678-703. Participation de Michel Foucault à une discussion sur le travail social et sa clientèle. Les autres participants étaient : Jean-Marie Domenach, Jacques Donzelot, Jacques Julliard, Philippe Meyer, Rene Pucheu, Paul Thibaud, Jean-René Treanton, Paul Virilio.

"Médecine et lutte de classes ", La Nef 49 (octobre/décembre 1972), p. 1-4. Table ronde entre Michel Foucault et les membres du Groupe Information Santé. 
«The Guillotine Lives», traduit sous forme abrégée par Paul Auster, The New York Times (8 avril 1973), section 4, p. 15.

«Histoire des systèmes de pensée», Annuaire du Collège de France 72 (1972), p. 283286. Résumé đ'un cours donné au Collège de France en 1972. Titre du cours: Théories et institutions pénales. Séminaire: Psychiatrie et pénalité au XIX siècle. Réédité en annexe dans Kremer-Marietti, Michel Foucault, p. 201-205.

Préface au livre de Serge Livrozet, De la prison d la révolte, Paris, Mercure de France, 1973, p. 7-14.

Présentation. À moi, Pierre Rivière, ayant égorgé ma mère, ma saur et mon frère. Édité sous la direction de Michel Foucault. Paris, Gallimard, Julliard, 1973, p. 9-15.

«L'intellectuel serı à rassembler les idées, mais... son savoir est partiel par rapport au savoir ouvrier", Libération 16 (26 mai 1973), p. 2-3. Une conversation entre Michel Foucault et un ouvrier nommé José.

«Entretien avec Michel Foucault: à propos de l'enfermement penitentiaire *, Pro Justicia: Revue politique du droit 3-4 (octobre 1973), p.5-14. Une entrevue de A. Krywin et F. Ringelheim.

«Gefangnisse und Gefangnisrevolten», Dokumente: Zeitschrift für ubernationale Zusammenarbeit 29 (juin 1973), p. 133-137. Une entrevue de Bodo Morawe.

«Convoqués à la P.J», Le Nouvel Observateur 468 (29 octobre 1973), p.53. Écrit en collaboration avec Alain Landau et Jean-Yves Petit.

«Histoire des systèmes de pensée», Annuaire du Collège de France 73 (1973), p. 255267. Résumé d'un cours donné au Collège de France en 1973. Titre du cours: La société punitive. Séminaire: Pierre Rivière et ses œuvres. Rédité en annexe dans Kremer-Marietli, Michel Foucault, p. 206-221.

«Sexualitê et politique», Combat 9274 (27-28 avril 1974), p. 16.

Table ronde sur l'expertise psychiatrique. Actes: Cahiers d'action juridique 5-6 (décembre 1974 à janvier 1975). Republié dans Actes: Délinquances et ordre, Paris, Maspero, 1978, p. 213-228. Les autres participants étaient: Y. Bastie, A. Bompart, M. Diederichs, F. Domenach, P.Gay, J. Hassoun, J. Lafon, C. Marechal, P. Sphyras, F. Tirlocq. Les interventions de Michel Foucault ont été recueillies et publiées sous le titre «L'expertise psychiatrique», dans Actes 54: «La gouvernementalité », (êté 1986), p. 68.

«Histoire des systèmes de pensée», Annuaire du Collège de France 74 (1974), p. 293300. Résumé d'un cours donné au Collège de France en 1974. Titre du cours : Le pouvoir psychiatrique. Séminaire: Explication des textes médicaux et juridiques du XIX ${ }^{e}$ siècle.

Préface au livre de Bruce Jackson, Leurs prisons, Paris, Plon, 1975, p.i-vi.

«Des supplices aux cellules», Le Monde 9363 (21 février 1975), p. 16. Roger Pol Droit s'entretient avec Michel Foucault.

«Entretien sur la prison», Magazine littéraire 101 (juin 1975), p. 27-33. Une entrevue de J.J. Brochier. 
«Prison Talk: an interview with Michel Foucault», traduit par Colin Gordon. Radical Philosophy 16 (printemps 1977), p.10-15. Rédition dans Power/Knowledge, p. 37-54.

«Pouvoir et corps. Une entrevue avec Michel Foucault», dans Quel corps? 2 (1975). Republie dans une collection de textes sous le titre Quel corps ?, Paris, François Maspero, 1978, p.27-35.

«La machine à penser s'est-elle détraquée?», Le Monde diplomatique 256 (juillet 1975), p. 18-21. Citations de Michel Foucault parmi d'autres dans une enquête sur le thème «La pensée en crise» faite par Maurice Maschino.

"Aller à Madrid", Libération 538 (24 septembre 1975), p. 1-7. Une entrevue de Pierre Benoit concernant la délégation de sept intellectuels français, dont Michel Foucault, qui ont été expatriés de Madrid alors qu'ils dénoncaient les sentences de mort imposées aux militants anti-franquistes.

"Histoire des systèmes de pensée", Annuaire du Collège de France 75 (1975), p. 335339. Resumé d'un cours donné au Collège de France en 1975. Titre du cours: Les anormaux. Séminaire: L’expertise médico-légale en matière psychiatrique.

«Une mort inacceptable», préface au livre de Bernard Cuau, L'affaire Mirval ou Comment le récit abolit le crime, Paris, Les presses d'aujourd'hui, 1976, p. vii-xi.

«La politique de la santé au XVIII siècle», introduction à la première partie «L'institution hospitalière au XVIII ${ }^{e}$ siècle» d'une étude en trois parties faite sous la direction de Michel Foucault et publiée sous le titre Généalogie des équipements de normalisation: Les équipements sanitaires, Fontenay-sur-Bois, Centre d'études, de recherches et de formation institutionnelles (CERFI), 1976, p. 1-11.

«Crimes et châtiments en U.R.S.S. et ailleurs », Le Nouvel Observateur 585 (26 janvier 1976), p. 34-37. Une entrevue avec K.S. Karol.

"The Politics of Crime", Partisan Review 43 (1976), p. 453-459.

"L'extension sociale de la norme», Politique hebdo 212 (mars 1976), p. 14-16. Une entrevue avec P. Werner sur le livre de Szasz, Fabriquer la folie.

«Histoire des systemes de pensée», Annuaire du Collège de France 76 (1976), p. 361366. Résumé d'un cours donné au Collège de France en 1976. Titre du cours: Il faut défendre la société. Séminaire: L'utilisation des techniques psychiatriques en matière pénale.

Préface du livre Les juges kaki de Mireille Debard et Jean-Luc Henning, Paris, Éditions Alain Moreau, 1977, p. 7-10. Publié également dans Le Monde 10214 (1-2 décernbre 1977), p. 15.

«La vie des hommes infâmes», Les Cahiers du Chemin 29 (15 janvier 1977), p. $12-29$.

«Pouvoir et stratégies», Les révoltes logiques 4 (hiver 1977), p. 89-97.

«Non au sexe roi », Le Nouvel Observateur 644 (12 mars 1977), p.92-130. Une entrevue de Bernard-Henri Levy.

«Power and Sex: An Interview with Michel Foucault», traduit par David Parent, Telos 32 (êté 1977), p. 152-161. 
"L'asile illimité», Le Nouvel Observateur 646 (28 mars 1977), p. 66-67. Un compte rendu du livre de Robert Castel L'ordre psychiatrique.

«L'angoisse de juger », Le Nouvel Observateur 655 (30 mai 1977), p.92-126. Un débat sur la peine de mort avec Robert Badinter et Jean Laplanche, édité par Catherine David.

«Le jeu de Michel Foucault», Ornicar? 10 (juillet 1977), p.62-93. Une discussion entre Michel Foucault et Alain Grosrichard, Gérard Wajeman, Jacques-Alain Miller, Guy Le Gaufey, Catherine Millot, Dominique Colas, Jocelyne Livi, Judith Miller.

«Enfermement, psychiatrie, prison », Change: La folie encerclée 32-33 (octobre 1977), p.76-110. Dialogue entre Michel Foucault, David Cooper, Victor Fainberg et Jean-Pierre Faye.

«L'évolution de la notion d'individu dangereux dans la psychiatrie légale», Déviance et société, V (1981), p.403-422.

«La vie: l'expérience et la science», Revue de métaphysique et de morale 90 (janvier/mars 1985), p. 3-14.

Introduction de la version anglaise: Herculine Barbin. Being the Recently Discovered Memoirs of a Nineteenth Century French Hermaphrodite. Traduit par Richard McDougall, New York, Pantheon Books, 1980, p. vii-xvii.

"Governmentality», traduit par Rosi Braidotti. Ideology and Consciousness 6 (automne 1979), p. 5-21.

Language, Counter-Memory, Practice. Essais et entrevues choisis. Avec une introduction de Donald F. Bouchard. Oxford, Basil Balckwell, 1978.

"La poussière et le nuage», L'impossible prison: Recherches sur le système pénitentiaire au XIXe siècle. Édité par Michelle Perrot. Paris, Seuil 1980, p. 29-39. Même s'il n'a pas été publié avant mai 1980, ce texte fut écrit en 1978 en réplique à un texte de Jacques Léonard publié dans le même document: "L'historien et le philosophe: À propos de: Surveiller et punir; naissance de la prison".

Postface. L'impossible prison (cf. $\left.\mathrm{n}^{\circ} 190\right)$, p. 316-318.

«Du pouvoir », L'Express 1722 (13 juillet 1984), p. 56-62. Une entrevue avec Michel Foucault, faite par Pierre Boncenne en juillet 1978, mais publiée seulement après la mort de Foucault.

«À quoi rêvent les Iraniens?», Le Nouvel Observateur 726 (9-16 octobre 1978), p. 48-49.

«Le citron et le lait *, Le Monde 10, 490 (21 octobre 1978), p. 14. Une recension du livre de Boucher Le ghetto judiciaire.

«Réponse de Michel Foucault à une lectrice iranienne", Le Nouvel Observateur 731 (13 novembre 1978 ), p. 26.

«Histoire des systèmes de pensê" \#, Annuaire du Collège de France 78 (1978), p. 445 449. Résumé d'un cours donné au Collège de France en 1978. Titre du cours: Sécurité, territoire et population. Séminaire: La médicalisation en France depuis le XIX siècle. 
«L'esprit d'un monde sans esprit », un entretien avec Michel Foucault par Claire Brière et Pierre Blanchet. Publié en annexe à leur livre Iran: la révolution au nom de Dieu, Paris, Seuil, 1979, p. 225-241.

Préface au livre de Peter Brückner et Alfred Krovoza, Ennemi de l'État, Claix, La pensée sauvage, 1979, p. 4-5.

«La phobie d'État», Liberation 967 (30 juin/1" juillet 1984), p. 21. Extrait d'un cours donné par Michel Foucault le 31 janvier 1979 au Collège de France.

"Michel Foucault et l'Iran *, Le Matin 647 (26 mars 1979), p. 15. Une courte réplique a un article qui déplorait sa position sur la révolution en Iran. Voir Claudie et Jacques Broyelle, "À quoi rêvent les philosophies?", Le Matin 646 (24 mars 1979), p. 13.

«Lettre ouverte à Mehdi Bazargan», Le Nouvel Observateur 753 (14 avril 1979), p. 46.

«Pour une morale de l'inconfort ", Le Nouvel Observateur 754 (23 avril 1979), p. 8283. Une recension de L'ère des ruptures de Jean Daniel.

«Inutile de se soulever?», Le Monde 10, 661 (11 mai 1979), p. 1-2.

"Omnes et Singulatim: Towards a criticism of "political reasons" », Conférence donnée à l'Université Stanford les 10 et 16 octobre 1979 in Sterling McMurrin (ed) The Tanner Lectures on Human Values II (1981), Salt Lake City, University of Utah Press, 1981, p. 225-254.

"Omnes et Singulatim: vers une critique de la raison politique", traduit par P.E. Dauzat. Le débat 41 (septembre et novembre 1986), p.5-35.

«Luttes autour des prisons», Esprit 35 (novembre 1979), p. 102-111. Discussion entre Michel Foucault (sous le pseudomyne de Louis Appert), Antoine Lazarus et François Colcombet sur les changements survenus dans les prisons dans les années 1970.

"Histoire des systèmes de pensée», Annuaire du Collège de France 79 (1979), p. $367-$ 372. Résumé d'un cours donné au Collège de France en 1979. Titre du cours: Naissance de la biopolitique. Séminaire: Problèmes de méthode en histoire des idées.

Préface du livre de Roger Knobelspiess, Q.H.S.: Quartier de haute sécurité, Paris, Stock, 1980, p.11-16 (31 mars 1980).

"Le philosophe masqué, Le Monde Dimanche 10, 945 (6 avril 1980), p.I et p.XVII. Une entrevue de Christian Delacampagne et publiée une première fois sans identifier Foucault.

Power/Knowledge, C. Gordon (éd.), New York, Harvester Press, 1980.

"Sexuality and Solitude», London Review of Books 3 (21 mai/3 juin 1981), p.5-6. Le texte d'une conférence de Foucault donnée le 20 novembre 1980 au New York Institute for the Humanities. Ce texte fut par la suite publie dans Humanities in Review I (1982), édité par David Rieff, New York, Cambridge University Press, 1982, p. 3-21.

«Le vrai sexe », Arcadie 27 (novembre 1980), p. 617-625. 
«De l'amitié comme mode de vie „, Le Gai Pied 25 (avril 1981), p.38-39.

"L'intellectuel et les pouvoirs", La Revue Nouvelle 80 (1984), p. 338-345. Entrevue de Christian Panier et Pierre Watté, le 14 mai 1981.

« Est-il donc important de penser?», Libération (30-31 mai 1981), p. 21. Entrevue avec Michel Foucault faite par Didier Eribon.

«Face aux gouvernements, les droits de l'Homme», Libération 967 (30 juin/1" juillet 1984), p. 22. Une déclaration de Michel Foucault faite en juin 1981, mais publiée seulement après sa mort, au sujet des «boat people» de l'Asie du sud-est.

«Il faut tout repenser la loi et la prison*, Libération 45 (6 juillet 1981), p. 2. Sur la grève de la faim par les détenus.

«De la nécessité de mettre un terme à toute peine», Libération 108 (18 septembre 1981 , p. 5.

«Les réponses de Pierre Vidal-Naquet et de Michel Foucault», Libération 185 (18 décembre 1981), p. 12. À propos de la réaction du gouvernement français sur l'imposition de la loi martiale en Pologne.

Le désordre des familles: Lettres de cachet des Archives de la Bastille. Une série de documents de la police, publiée avec des introductions de Michel Foucault et d'Arlette Farge, Paris, Gallimard/Julliard, 1982.

«Le combat de la chasteté», Communications 35 (mai 1982), p. 15-25.

Entrevue. Ethos I, 2 (automne 1983), p. 4-9. Faite par Stephen Riggins le 22 juin 1982.

"Que fabriquent donc les hommes ensemble?», Le Nouvel Observateur 1098 (22-28 novembre 1985), p. 54-55.

«Le terrorisme ici et là», Libération (3 septembre 1982), p.12. Discussion avec D. Eribon.

«L'âge d'or de la lettre de cachet », L'Express 1638 (3 décembre 1982), p. 35-36. Une entrevue avec Michel Foucault et Arlette Farge, faite par Yves Hersant sur Le désordre des familles, citế plus haut.

«Un système fini face à une demande infinie», in Sécurité sociale: l'enjeu. Paris, Éditions Syros, 1983, p. 39-63. Une entrevue avec R. Bono.

«Structuralism and Post-Structuralism: An Interview with Michel Foucault », Telos 55 (printemps 1983), p. 195-211. Une entrevue faite par Gérard Raulet et traduite par Jeremy Harding. Publiée d'abord en Allemand sous le titre «Um welchen Preis sagt die Vernunft die Wabrheit? » Spuren 1-2 (1983).

"Politics and Ethics: An Interview", traduit par Catherine Porter, in The Foucault Reader, sous la direction de Paul Rabinow, New York, Pantheon Books, 1984, p. 373-380. Entrevues faites en avril 1983 par Paul Rabinow, Charles Taylor, Martin Jay, Richard Rorty et Leo Lowenthal.

"On the Genealogy of Ethics: An Overview of Work in Progress", in Michel Foucault: Beyond Structuralism and Hermeneutics, Chicago, University of Chicago Press, 1983, $2^{\mathrm{e}}$ édition, p. 229-252. Une entrevue faite par Hubert Dreyfus et 
Paul Rabinow. Une traduction abrégée faite par Jacques B. Hess a été publiée en français sous le titre «Le sexe comme une morale», Le Nouvel Observateur (1 ${ }^{\text {e }}$ juin 1984), p.62-66.

«The subject and Power», postface au précédent ouvrage, Michel Foucault: Beyond Structuralism and Hermeneutics, p. 208-226.

"Qu'appelle-t-on punir?", Revue de l'Université de Bruxelles (1984): Punir mon bon souci, pour une raison pénale, p.35-46. Une entrevue avec Foulek Ringelheim, faite en décembre 1983.

"L'éthique du souci de soi comme pratique de liberté", Concordia 6 (1984), p.99-116. Une entrevue avec Raul Fomet-Betancourt, Helmut Becker et Alfredo GomezMüller, faite le 20 janvier 1984.

"A Last Interview with French Philosopher Michel Foucault", City Paper 8, 30 (27 juillet/2 août 1984), p. 18. Faite par Jamin Raskin en mars 1984.

«Interview de Michel Foucault», Actes 45-46 (1984): La prison autrement?, p. 3-6. Entrevue de Catherine Baker en avril 1984.

«Pour en finir avec les mensonges», Le Nouvel Observateur 1076 (21-27 juin 1985), p. 76-77. Entrevue de Didier Eribon.

«Le retour de la morale », Les Nouvelles 2937 (28 juin/5 juillet 1984), p. 36-41. Entrevue faite par Gilles Barbedette et André Scala. Produite le 29 mai 1984, c'est probablement la dernière entrevue que Michel Foucault a donnée.

«FinaI Interview», traduite par Thomas Levin et Isabelle Lorenz, Raritan V, 1 (été 1985), p. 1-13.

«Création d'un Groupe d'information sur les prisons », Esprit 401 (mars 1971), p.531532. Co-signé par Michel Foucault, Jean-Marie Domenach et Pierre VidalNaquet.

«Power and Norm: Notes», notes d'une conférence donnée au Collège de France le 28 mars 1973. Traduit par W. Suchting dans Power, Truth, Strategy, p. 59-66.

De la Gouvernementalité, Paris, Seuil, 1989.

Résumés de cours 1940-1982. (Conférences, essais et leçons du collège de France), Paris, Julliard, 1989.

Michel Foucault: Politics, Philosophy, Culture, Interviews and other writings, 19771984, New York, Routledge Chapman and Hall, 1988. 


\section{B. QUELQUES ÉCRITS À PROPOS DE MICHEL FOUCAULT ET DE SON GEUVRE}

BERNAUER, J.W., Michel Foucault's Force of Flight, Toward an Ethics for Thought, Atlantic Highlands, N.J., Humanities Press International Inc., 1989.

BRIĖRE, Claire et Michel BLANCHET, Iran: La révolution au nom de Dieu, Paris, Seuil, 1979. Ce livre contient un entretien avec Michel Foucault que la volonté de voir et de comprendre a amené en Iran.

DELEUZE, Gilles, Foucault, Paris, Minuit, 1986.

DERRIDA, Jacques, "Cogito et histoire de la folie», Revue de métaphysique et de morale, 68,1963 , pp. $460-494$.

DREYFUS, H.L. et Paul RABINOW, Michel Foucault. Beyond Structuralism and Hermeneutics, avec une postface de Michel Foucault, Chicago, Chicago University Press, $2^{e}$ édition, 1983.

DREYFUS, H.L. et Paul RABINOW, Michel Foucault, un parcours philosophqiue: au-delà de l'objectivité et de la subjectivité, avec un entretien et deux essais de Michel Foucault, Paris, Gallimard, 1984.

ERIBON, Didier, Michel Foucault, Paris, Flammarion, 1989.

FLAHERTY, Peter, «(Con)textual contest: Derrida and Foucault on Madness and Cartesian Subject », Philosophy of the Social Sciences, 16, 2, 1986, p. 157-176.

GARLAND, P., «Foucault's Discipline and Punish. An exposition and Critique», in American Bar Foundation Research Journal, 1986, p. 847-881.

HABERMAS, J., «Taking Aim at the Heart of the Present: On Foucault's lecture on Kant's What is Enlightment» in Habermas, J., The New Conservatism, Cambridge, MA, Cambridge U.P., 1989.

HABERMAS, J., Der philosophische Diskurs des Moderne, Frankfurt am Main, Suhrkamp Verlag, 1985. (Cet ouvrage a été traduit en français sous le titre Le discours philosophique de la modernité, Paris, Gallimard, 1988).

HOY, David (ed.), Foucault. A Critical Reader, Basil Blackwell, 1986.

KURZWEIL, E., The Age of Structuralism: Levi-Strauss to Foucault, Columbia University Press, 1980.

Michel Foucault, numéro spécial de Libération, samedi 30 juin et dimanche $1^{\text {t }}$ juillet 1984.

Michel Foucault philosophe, rencontre internationale, Paris 9-11 janvier 1988. Collectif, coll. « Des travaux», Paris, Seuil, 1989.

ROUDINESCO, Elizabeth et al., Penser la folie: Essais sur Michel Foucault, Paris, Galilee, 1992.

STURROCK, J., Structuralism and Since: From Levi-Strauss to Derrida, Oxford University Press, 1979. 